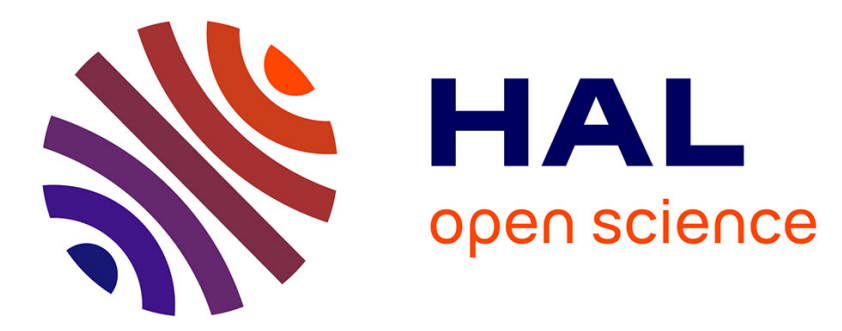

\title{
La philanthropie américaine et l'Europe: contribution à une histoire transnationale de l'américanisation
}

\author{
Ludovic Tournès
}

\section{To cite this version:}

Ludovic Tournès. La philanthropie américaine et l'Europe: contribution à une histoire transnationale de l'américanisation. Bulletin de l'Institut Pierre Renouvin, 2010, 31, http://ipr.univparis1.fr/spip.php?article487. halshs-00651974

\section{HAL Id: halshs-00651974 \\ https://shs.hal.science/halshs-00651974}

Submitted on 14 Dec 2011

HAL is a multi-disciplinary open access archive for the deposit and dissemination of scientific research documents, whether they are published or not. The documents may come from teaching and research institutions in France or abroad, or from public or private research centers.
L'archive ouverte pluridisciplinaire HAL, est destinée au dépôt et à la diffusion de documents scientifiques de niveau recherche, publiés ou non, émanant des établissements d'enseignement et de recherche français ou étrangers, des laboratoires publics ou privés. 


\section{Ludovic Tournès \\ La philanthropie américaine et l'Europe : contribution à une histoire transnationale de l'américanisation.}

Habilitation à diriger des recherches (sous la direction de Robert Frank), université Paris I-Panthéon Sorbonne. Soutenue le 22 novembre 2008 devant un jury composé de : Volker Berghahn (Columbia University), Robert Frank, université ParisI Panthéon-Sorbonne), Giuliana Gemelli (université de Bologne), Nancy Green (Ecole des Hautes Etudes en Sciences Sociales), Pascal Ory (université Paris-I Panthéon-Sorbonne), Jean-François Sirinelli (Institut d'Etudes Politiques).

Ce travail présente une recherche inédite portant sur les activités internationales des grandes fondations philanthropiques américaines, un domaine dont l'historiographie, très riche, est presque exclusivement en langue anglaise. II confronte également les résultats de cette recherche empirique avec une réflexion épistémologique sur le concept d'américanisation. Enfin, il ouvre des perspectives futures pour la recherche individuelle et collective. Le mémoire de recherche proprement dit s'appuie sur une bibliographie importante et sur le dépouillement de 36 fonds d'archives très divers : archives de la fondation Rockefeller et du Carnegie Endowment for International Peace; Archives nationales françaises ; archives de la Société des Nations; de l'Institut International de Coopération Intellectuelle; du Département d'Etat américain ; d'institutions publiques (Collège de France, Centre National de la Recherche Scientifique) ou privées (Institut Pasteur) ; fonds privés ; etc. II comprend trois parties. La première, historiographique et épistémologique, présente un bilan problématique de la production scientifique relative aux activités internationales de la philanthropie américaine (chapitre I), et une discussion sur le concept d'américanisation (chapitre II). Les parties suivantes sont consacrées à des travaux empiriques: la deuxième détaille l'ensemble des actions menées par la fondation Rockefeller en France avant 1939 (chapitres III à VIII); la troisième s'intéresse à l'implication du Carnegie Endowment for International Peace et de la fondation Rockefeller dans les activités de la Société des Nations au cours de l'entredeuX-guerres (chapitres IX à XII).

\section{L'historiographie de la philanthropie}

L'historiographie des activités internationales des fondations philanthropiques américaines est très riche; elle $a$, jusqu'à une date récente, été dominée par les chercheurs américains et anglais et reste encore peu connue en France, où la philanthropie ne constitue pas un domaine d'études à part entière. Si les chercheurs français se sont intéressés à la " philanthropie ", c'est dans une acception du terme plus restreinte qu'aux États-Unis, les études existantes portant essentiellement sur les associations caritatives, et ayant été réalisées le plus souvent par des historiens de la protection sociale. 
L'historiographie de la philanthropie américaine s'est, pour une large part, structurée autour d'une opposition entre un courant que l'on qualifiera de libéral, mettant en avant les réalisations des fondations, et un courant gramscien insistant sur leur rôle dans la reproduction de l'ordre capitaliste. Les travaux portant sur les activités internationales des fondations n'ont pas échappé à ce clivage, et l'on pourrait même soutenir qu'il y a été encore plus opératoire que dans les études relatives à leur politique domestique, en raison de la montée en puissance des ÉtatsUnis sur la scène géopolitique tout au long du XXe siècle. Les deux courants, soustendus par des options idéologiques différentes, ont donné lieu à une production importante jalonnée de polémiques encore actives dont les grandes lignes ont été retracées afin d'en présenter les apports respectifs tout en soulignant leurs limites et leurs lacunes, non pas pour prendre partie pour l'une ou l'autre, mais pour ouvrir à l'historiographie de nouvelles pistes que ce clivage a pu contribuer à occulter. En particulier, le paradigme longtemps dominant dans l'histoire de la philanthropie tend peu à peu à se déplacer du donateur vers le récipiendaire, mais aussi, et c'est sans doute plus important, vers les interactions qui existent entre les deux.

II se dégage en effet des nombreux travaux menés depuis la fin des années 1990 une tendance à insérer les fondations dans un tissu de circulations transnationales qui constitue une direction de recherches féconde. C'est elle qui est développée dans ce mémoire : l'hypothèse qui lui sert de fil conducteur consiste à considérer les projets des fondations non pas comme pas des constructions internes élaborées dans le huis clos des Board of Trustees mais bien plutôt comme des constructions transnationales consécutives aux multiples négociations menées simultanément par les officers dans de nombreux pays. Elle s'inscrit en faux contre une perspective interprétant l'action des les États-Unis en terme d'exportation ou de diffusion unilatérale, considérant plutôt que l'action des fondations à l'étranger consiste autant à "diffuser " dans le monde un "modèle " américain qu'à aller chercher des " modèles » dans les différents pays où elles interviennent pour les appliquer aux États-Unis. Dans cette perspective, le « modèle » américain dont les fondations seraient porteuses constitue à bien des égards une synthèse, réalisée empiriquement, de la multitude d'expériences internationales rencontrées par les philanthropes dans leurs secteurs d'activité. On perçoit dès lors que c'est toute la vision traditionnelle de "l'américanisation » qui s'en trouve affectée, la notion de " modèle " américain étant alors la résultante non d'un phénomène de diffusion unillatérale, mais d'un processus complexe d'import-export. Dans cette perspective, les fondations ne sont pas tant des vecteurs de diffusion que des plaques tournantes contribuant à la construction de savoirs et de pratiques, mais aussi à leur redistribution, depuis les États-Unis vers le reste du monde, mais aussi en sens inverse. On voit dans ces conditions tout l'intérêt que peut revêtir une histoire transnationale des fondations philanthropiques.

\section{Une démarche transversale}

En raison de mon parcours scientifique d'historien du culturel, j'ai abordé l'histoire des fondations avec une perspective différente de la plupart des spécialistes de la philanthropie. L'originalité de mon approche se manifeste de deux manières. La première réside dans l'approche globale de l'action d'une fondation dans un pays donné (la France), alors que la plupart des études concernent des actions 
sectorielles et souvent limitée dans le temps. Cette approche a permis de mettre en lumière les connexions qui existent entre les différents domaines d'intervention de la philanthropie rockefellerienne : elle ne s'est pas réduite à une série d'études de cas, mais a tenté de cerner la globalité du projet rockefellerien pour la France et l'unité de son action tous secteurs confondus: ont été ainsi analysés successivement l'ensemble des secteurs dans lesquels la fondation Rockefeller intervient en France au cours de l'entre-deux-guerres: la santé publique, l'enseignement supérieur, la recherche scientifique dans le domaine biomédical et dans celui des sciences sociales.

Cette démarche transversale a également été mise en œuvre à l'échelle internationale, dans l'étude des relations entre les fondations Carnegie et Rockefeller et la Société des Nations. Ici encore, l'objectif a consisté à passer par-dessus les frontières des sous-disciplines pour étudier l'action des fondations dans tous les domaines en s'intéressant aux trois sections de la SDN bénéficiant du financement philanthropique: la Section d'Hygiène, I'Organisation Economique et Financière, I'Institut International de Coopération Intellectuelle. Cet élargissement de la perspective a permis d'aller plus loin dans l'analyse du projet intellectuel philanthropique qui n'apparaissait que partiellement à travers l'étude de cas française. Elle a permis d'acquérir une vision claire du projet de la philanthropie américaine vis-à-vis de la Société des Nations, alors que son investissement dans cette organisation était en contradiction, au moins apparente, avec le retrait du gouvernement américain des affaires européennes adoptée au lendemain de la Première guerre mondiale. Elle a notamment mis en évidence l'ambition nourrie par les grandes fondations de contribuer à la construction d'un ordre international en participant aux activités de la première organisation internationale multilatérale de l'histoire de l'humanité. Elle a également permis d'approfondir la compréhension de la méthode philanthropique, à travers notamment la contribution des fondations à l'élaboration d'une expertise internationale. L'un des apports de cette analyse à I'histoire des relations internationales est d'avoir montré que les deux fondations, grâce à leurs disponibilités financières et leur capacité à mobiliser des réseaux internationaux, établissent leur hégémonie intellectuelle sur les travaux développés par la Société des Nations dans certains secteurs, en particulier le domaine de l'expertise économique internationale, témoignant ainsi de la profonde implication des Etats-Unis dans la SDN, fut-ce par l'intermédiaire d'organisations non gouvernementales.

Outre l'approche globale de la politique philanthropique, la deuxième originalité de ce travail réside dans la focalisation de l'étude sur les boursiers Rockefeller, ces fellows que l'on trouvera tout au long des chapitres III à XII du volume 2. Alors que la plupart des historiens de la philanthropie se sont consacrés avant tout à une approche institutionnelle des actions des fondations, je me suis intéressé de près aux individus qui bénéficient des financements des fondations. Cette approche a permis de mettre en évidence le tissu de relations dans lesquelles elles s'inscrivent et que leurs actions suscitent, de sorte que ce travail n'est pas tant consacré aux fondations per se qu'aux circulations transnationales qui s'organisent autour d'elles. On y trouvera donc de nombreux développements portant sur les négociations préalables aux financements, les voyages permanents des officers des fondations, leur insertion parfois profonde dans les réseaux politiques et intellectuels locaux, ou encore leur stratégie visant à organiser une circulation systématique de l'information et des chercheurs à l'échelle mondiale, un processus dont le programme fellowships de la fondation Rockefeller est sans doute l'illustration la plus 
emblématique; avec ses quelques 6000 bénéficiaires dans le monde entier au cours de l'entre-deux-guerres, il constitue en effet à lui seul un pan de l'histoire des échanges scientifiques internationaux pendant cette période. L'étude des réseaux, des voyages et des connexions organisées par les fondations a été menée à l'échelle nationale, à travers l'étude du cas français, mais aussi à l'échelle internationale : de ce point de vue, l'analyse des relations entre trois organisations internationales (la fondation Rockefeller, le Carnegie Endowment for International Peace et la Société des Nations) a permis de mettre en avant une dimension transnationale que l'étude du cas français ne permettait que d'entrevoir. C'est également ici que s'est manifestée la spécificité de mon approche par rapport aux historiens de la philanthropie, l'étude des itinéraires des fellows ayant été un moyen d'évaluer l'impact des actions de la fondation Rockefeller en France sur le long terme de manière plus fine que le simple examen des institutions financées, qui ne dit pas grand-chose de leur inscription dans le paysage intellectuel et institutionnel.

\section{Repenser l'américanisation}

La première partie du mémoire de recherche a été consacrée à une discussion critique du concept d'américanisation. Le fil conducteur de cette discussion critique est l'insatisfaction ressentie devant le paradigme diffusionniste qui a longtemps constitué le cadre de réfléxion, implicite ou explicite, des travaux sur l'américanisation, un cadre dont l'analyse des circulations multiples auxquelles les fondations participent montre non seulement les limites, mais aussi les impasses. Ce constat m'a amené à mettre en évidence des pistes de recherches ébauchées dans des travaux européens et américains récents, dont le point commun est d'amorcer une remise en cause de ce paradigme qui doit, à mon sens, être poursuivie. C'est sur ce socle historiographico-épistémologique que s'appuient les deux parties suivantes, qui entendent illustrer une approche non diffusionniste des processus d'américanisation et contribuer, comme l'indique le titre du mémoire, à l'écriture d'une histoire transnationale de l'américanisation. Pour cela, j'ai choisi de concentrer mon propos sur l'entre-deux-guerres, période en général délaissée par les historiens de l'américanisation au profit de l'après 1945.

Pour clarifier le débat, j'ai d'abord analysé la généalogie intellectuelle du terme " américanisation », ce qui a permis mettre en évidence la congruence qui s'est opérée entre trois notions devenues progressivement synonymes sous la plume des chercheurs : américanisation, acculturation et modernisation. J'ai ensuite tenté de mettre en lumière deux apories majeures de la notion d'américanisation telle qu'elle est employée par la plupart des historiens. La première consiste à la considérer comme un phénomène d'acculturation et à la penser dans une perspective diffusionniste qui ne permet pas de la comprendre dans toute sa complexité. En effet, l'interprétation diffusionniste constitue de facto les États-Unis en émetteur imperméable aux sollicitations extérieures, et les pays confrontés à leur puissance en autant de récepteurs, certes actifs dans leurs réinterprétations, mais dont les études des "réponses » données aux sollicitations américaines ne mettent pas en valeur les dynamiques propres. L'autre aporie est la perspective bilatérale selon laquelle sont organisées la plupart des études, sous forme d'autant de monographies consacrées aux face-à-face entre les États-Unis et les pays confrontés à leur 
puissance, une perspective qui, malgré ses évidentes justifications, aboutit malgré à tout à enfermer l'analyse de l'échange dans un cadre trop étroit pour refléter sa complexité. Pour surmonter ces deux apories auxquelles se sont heurtées la plupart des études sur l'américanisation (y compris celles menées par l'auteur de ces lignes), il importe de clarifier des directions de réflexion qui ont émergé dans des travaux récents, et, en s'appuyant notamment sur les acquis des différents courants de l'histoire transnationale en plein développement depuis la décennie 1990, de proposer une perspective épistémologique permettant de repenser le phénomène d'américanisation. Cette perspective tient en deux mots: circulations et multilatéralisme. Circulations d'abord, car il paraît nécessaire que l'analyse, longtemps focalisée sur les questions de réception, s'intéresse désormais aux passages et à leur rôle structurant dans la mise en place de la puissance américaine ; mais aussi, et peut-être surtout, parce que la perspective diffusionniste à sens unique doit à l'évidence être abandonnée au profit d'une étude à double sens dans laquelle les États-Unis et les autres sont à la fois, et en même temps, émetteurs et récepteurs. Multilatéralisme ensuite, car de telles circulations sont à l'évidence loin de se limiter à deux partenaires : par le jeu des passages transnationaux, des allersretours et des détours, c'est en réalité de multiples pays qu'elles concernent.

\section{L'apport des études de cas}

Les acquis des différentes études de cas menées dans les chapitres successifs ont permis de dégager quelques directions de réflexion relatives à la question de l'américanisation.

La première remarque qui s'impose est la place importante tenue par la philanthropie dans le paysage des relations internationales dès l'entre-deux-guerres, son inscription dans un tissu transnational de circulations et de réseaux lui permettant d'articuler un projet global d'ampleur mondiale et un ensemble de projets relatifs à des aires nationales spécifiques. En effet, le cas de la Rockefeller, et dans une moindre mesure celui du Carnegie Endowment, montrent que les grandes fondations sont capables de se projeter dans n'importe quel pays et d'y entreprendre des actions de terrain dans des secteurs variés (prophylaxie, enseignement de la médecine ou des soins infirmiers, recherche biomédicale, recherche en sciences sociales, etc.). D'autre part, ces organisations sont à la fois des bailleuses de fonds, mais aussi des partenaires actives des processsus engagés, établissant un contact très proche avec le terrain et suivant de près les projets mis en œuvre, pendant parfois très longtemps. Dans ce processus, il est frappant de constater à quel point l'organisation philanthropique a la capacité de penser simultanément à l'échelle mondiale, et à l'échelle nationale et même locale. L'exemple de la fondation Rockefeller est à cet égard éclairant : entre l'aide financière à la Section d'Hygiène de la Société des Nations, la subvention de l'Office National d'Hygiène Sociale destiné à soutenir la coordination de la politique française de santé publique, la subvention de l'école d'infirmières de Lyon ou encore le soutien à l'établissement d'un réseaux de dispensaires dans le département de l'Hérault, le travail simultané à plusieurs échelles est constitutif de la méthode Rockefeller.

L'articulation entre les dimensions locale, nationale et internationale est essentiellement le fait de trois catégories d'acteurs: les trustees, les officers et les fellows. Les premiers assurent la définition des grandes orientations de la fondation, 
et votent les budgets alloués chaque année aux différents projets; les seconds voyagent en permanence dans le monde entier, détectent des terrains d'intervention potentiels, conçoivent des projets et assurent la circulation des informations entre les différents organismes subventionnés. Les troisièmes sont des agents majeurs de transferts de savoir et de pratiques scientifiques d'un pays à l'autre. Appuyées sur leurs disponibilités financières et sur une pratique très poussée de la constitution et de l'expansion de réseaux, les fondations américaines élaborent ainsi une diplomatie spécifique et s'imposent dès l'entre-deux-guerres comme des actrices incontournables dans le domaine de l'organisation des échanges scientifiques internationaux, en dialoguant concurremment avec des institutions publiques ou privées, des administrations locales, nationales ou des organisations internationales. Elles s'imposent comme des plaques tournantes dans la construction et la circulation des savoirs et des pratiques à l'échelle internationale, jouant un rôle important dans le basculement des équilibres intellectuels mondiaux au profit des Etats-Unis, et ce, dès avant la deuxième guerre mondiale.

C'est bien cette fonction de plaque tournante assurée par les fondations, ainsi que leur politique internationale fondée sur la création de réseaux, qui vient enrichir la problématique de l'américanisation en montrant que le paradigme diffusionniste est insuffisamment explicatif, et que la présence et l'influence américaine en Europe au XXe siècle, indéniablement fortes, ne se présentent sans doute pratiquement jamais sous la forme d'une diffusion unilatérale ni unidirectionnelle mais empruntent des circuits complexes dans lesquels les Etats-Unis eux-mêmes sont impliqués. C'est particulièrement net pour l'étude portant sur la Rockefeller en France.

Celle-ci, en effet, n'apporte rien directement dans l'Hexagone: sa stratégie consiste avant tout à se greffer sur des projets en cours et à s'introduire au coeur des réseaux personnels et institutionnels existants pour peser sur l'orientation des projets déjà entamés et donner, le cas échéant, une impulsion parfois décisive en agissant dans un domaine bien identifié ou en coordonnant des activités séparées avant son arrivée. L'analyse des négociations, parfois très longues, qui précèdent les actions engagées, a permis aussi de montrer comment les projets mis en œuvre par la fondation étaient largement coproduits sur le moment même avec les interlocuteurs locaux ayant leur dynamique propre, plutôt que réinterprétés ultérieurement, comme le suggèrent le plus souvent les approches diffusionnistes de l'américanisation. Par ailleurs, l'insertion profonde de la fondation Rockefeller dans les réseaux universitaires et politico-administratifs français de l'entre-deux-guerres, lui permet souvent, bien que son statut d'organisation américaine ne disparaisse jamais, de se faire reconnaître comme une partenaire à part entière des projets engagés : ce fait majeur interdit de penser le processus d'américanisation en termes de face à face global entre deux cultures essentialisées.

Dans ce processus, il n'y a donc pas tant une exportation directe de méthodes américaines qu'une imbrication avec les multiples expériences que mène en même temps la fondation dans différents pays du monde, et où il serait sans doute vain de démêler ce qui provient des Etats-Unis, de la Chine, du Brésil ou de la Tchécoslovaquie. Ce que la Rockefeller apporte en France ne constitue jamais une exportation directe et "brute " de méthodes venues des Etats-Unis, mais la version américanisée de formes empruntées ailleurs et introduites en Fance par un circuit indirect et complexe. C'est le cas du full-time system des faculté de médecine, qui, s'il fallait absolument le caractériser en termes nationaux, serait bien plus " allemand » qu' " américain », la réforme flexnerienne des facultés de médecine américaines réalisée au cours des années 1910 et 1920 s'étant fondée 
majoritairement sur le " modèle » allemand, que l'action de la Rockefeller a consisté à tenter de réexporter en France via l'enclave " allemande " en territoire français que constitue la faculté de médecine de Strasbourg, sans doute la première en France à avoir mis en place un full time system avant la lettre. De ce point de vue, on pourrait presque dire que la dimension la plus importante du rôle de la Rockefeller en France après 1918 ne consiste pas tant à importer un système " américain » qu'à contribuer à rendre acceptable aux élites médicales françaises un système " allemand " en le parant de couleurs " américaines » qui lui enlèvent sa symbolique négative, laquelle constitue sans doute un frein à la réforme de l'enseignement médical français en raison des réticences d'une partie du milieu médical à adopter le modèle de la médecine scientifique dont les facultés d'outre-Rhin sont les principales porteuses. $\mathrm{Vu}$ sous cet angle, le processus d'américanisation ne consisterait pas à " apporter » en Fance des savoirs et des pratiques américaines, mais plutôt à inscrire la France dans une dynamique transnationale d'échanges de savoirs et de pratiques que les fondations tentent de créer, d'organiser et de contrôler à l'aide de leur réseaux.

C'est un processus similaire que l'on peut observer, à l'échelle internationale, dans le cas de la Société des Nations, notamment à travers la manière dont la fondation Rockefeller promeut les recherches empirico-quantitatives à la Conférence Permanente des Hautes Etudes Internationales en y introduisant systématiquement ses fellows. La plupart d'entre eux sont européens, et si une partie importante est allé étudier aux Etats-Unis, ils n'y sont pas forcément allé " apprendre » des méthodes empirico-quantitatives déjà en plein développement dans plusieurs pays d'Europe, en particulier l'Autriche, les Pays-Bas ou la Norvège. De ce point de vue, gageons que ces fellows ont autant " appris " à leurs collègues américains qu'ils ont appris d'eux. Le phénomène est ici similaire à celui que l'on observe avec l'émigration des élites intellectuelles allemandes et autrichiennes aux Etats-Unis après la prise de pouvoir des nazis en Allemagne et en Autriche, cette émigration intellectuelle contribuant à féconder le système universitaire américain et à assurer sa montée en puissance au cours des années trente. Le développement des méthodes empirico-quantitatives au cours de cette période est sans doute loin d'être une invention américaine ; en revanche, il est sûr que la fondation Rockefeller joue un rôle majeur dans la redistribution à l'échelle du continent européen de travaux menés en Autriche ou en aux Pays-Bas, via un circuit complexe de circulations dont les principaux points nodaux sont la Conférence Permanente des Hautes Etudes Internationales et les multiples instituts d'études de la conjoncture économique européens financés par la fondation dans les années trente; quant aux fellowships, elles assurent le lien et la circulation des savoirs entre ces différentes institutions, ainsi qu'entre elles et les institutions américaines, qui profitent autant des savoirs élaborés en Europe qu'elles exportent des savoirs américains sur le vieux continent.

La fonction de plaque tournante exercée par la fondation Rockefeller prend ici toute sa dimension, car on la voit fonctionner à l'échelle européenne, et assurer le lien transatlantique avec les milieux universitaires américains. Dans cette perspective, le processus d'américanisation réside avant tout dans la manière dont une institution américaine comme la fondation Rockefeller se place au centre de réseaux préexistants qu'elle contribue à réorganiser et réorienter grâce à sa puissance financière et à sa stratégie visant également à créer des réseaux toujours en expansion. C'est ici que se situe l'apport majeur des historiographies transnationales au paradigme de l'américanisation, car ce sont elles qui ont mis en avant l'existence de ce continuum de circulations qui rend nécessaire une révision susbantielle de la problématique diffusionniste. L'épisode du déménagement du 
Département Financier, de l'Economie et des Transports de la SDN à Princeton en 1940 est emblématique de la limitation de cette problématique, car il montre on ne peut plus clairement qu'une partie importante du processus d'américanisation se déroule aux Etats-Unis même : au-delà de la dimension symbolique de l'épisode qui manifeste clairement un changement d'hégémonie, on assiste à l'introduction dans le paysage intellectuel américain d'une expertise d'origine européenne. Les économistes européens, et en particulier ceux du Département Financier, de l'Economie et des Transports, vont en effet participer activement au travail de réflexion engagé par les universitaires américains en vue de la construction de l'ordre économique international formalisé aux conférences de Dumbarton Oaks et de Bretton Woods. Traditionnellement considéré comme un ordre « américain » imposé au monde en 1944, celui-ci constitue bien plus, dans cette perspective, une construction transatlantique, et si elle acquièrt cette étiquette " américaine », c'est avant tout parce que le rapport de force géopolitique s'est radicalement renversé en faveur des Etats-Unis entre 1939 et 1945.

Au terme de ce panorama problématique, deux éléments semblent s'imposer. La première est qu'il est nécessaire de réviser les catégories d'analyse de l'américanisation et de recadrer la perspective adoptée jusqu'à présent. L'un des principaux apports de la florissante historiographie transnationale menée depuis les années 1990 est bien d'obliger à toiletter une bonne partie de l'arsenal lexical et conceptuel utilisé par la plupart des spécialistes de l'américanisation. Diffusion, réception, influence, acceptation, refus, réinterprétation, réponse : autant de termes qui ne suffisent pas à cerner la réalité du phénomène, sans parler du cadre national qui doit à l'évidence être élargi, même s'il reste une réalité prégnante que l'analyse ne peut ignorer.

La deuxième est qu'il ne semble pas pertinent d'interpréter le concept d'américanisation comme un processus de diffusion internationale d'une culture construite dans le huis clos des frontières étatsuniennes puis puis imposée à travers le monde grâce à ses qualités intrinsèques ou, selon l'angle adopté, par la force de l'impérialisme américain. II me semble plus justifier de l'interpréter comme la conquête par les Etats-Unis d'une position centrale dans un processus multilatéral d'unification et de normalisation internationale qui traverse un $X X e$ siècle où l'établissement de normes mondiales est devenu un enjeu de pouvoir non seulement économique, mais aussi géopolitique, intellectuel et culturel. Ce processus de construction se déroule autant en Europe qu'aux Etats-Unis, comme le montre l'intense activité des sections techniques de la Société des Nations ; mais les EtatsUnis, en grande partie du fait de l'activité des fondations privées, parviennent à en capter une partie importante et réussissent dans de nombreux cas à imposer leurs propres normes, en s'appuyant sur les activités de la SDN pour acquérir un leadership intellectel sur le processus de définition d'un ordre international qui s'opère au cours de l'entre-deux-guerres, avant que la nouvelle donne géopolitique issue de la Deuxième ne leur donne le poids et le prestige nécessaire pour apparaître comme l'origine unique de cet ensemble de normes dont la guerre froide va contribuer à construire la cohérence en réaction contre le " modèle " soviétique, et à qui elle va donner le statut d'un modèle de société proposé à l'Occident. Alors 
que les interventions des Américains en Irak en 1991 puis en 2003 ont peut-être achevé de casser l'image internationale de puissance démocratique et modernisatrice qu'ils avaient acquise après la Deuxième guerre mondiale, et alors que le demi-siècle à venir va voir la remise en cause en raison de l'hégémonie américaine en raison de l'émergence de nouvelles grandes puissances telles que la Chine qui s'apprêtent à contester l'hégémonie américaine, cette perspective permet de reconsidérer, et donc de mieux comprendre, la place des Etats-Unis dans le monde contemporain.

Ludovic Tournès, professeur à l'université Pars-Ouest Nanterre 